\title{
Inspección mecánico-visual de ampolletas farmacéuticas de vidrio mediante el uso de visión artificial y robótica
}

\section{Mechanical-visual inspection of pharmaceutical glass ampoules through the use of artificial and robotic visión}

GARCIA-GUTIEREZ, Felipe De Jesús*†, ROJAS-OLMEDO, Israel Alejandro, BARONGUADARRAMA, Alma y SANCHEZ-MANCILLA, Luis Fernando

Universidad Tecnológica del Valle de Toluca, Carretera del Departamento del D.F. km 7.5, Santa María Atarasquillo, Lerma, México

ID 1 ${ }^{\text {er }}$ Autor: Felipe De Jesús, Garcia-Gutierez / ORC ID: 0000-0003-4007-9430

ID $1^{\text {er }}$ Coautor: Israel Alejandro, Rojas-Olmedo / ORC ID: 0000-0003-3501-0084

ID $2^{\text {do }}$ Coautor: Alma, Baron- Guadarrama / ORC ID: 0000-0001-7898-7448

ID $3^{\text {er }}$ Coautor: Luis Fernando, Sanchez-Mancilla / ORC ID: 0000-0002-3400-7521

DOI: $10.35429 /$ JOIE.2019.10.3.1.4

Recibido Marzo 27, 2019; Aceptado Junio 30, 2019

\begin{abstract}
Resumen
Uno de los problemas que enfrenta actualmente la industria farmacéutica en un proceso automático, es asegurar que mediante una sólo inspección visual tradicional se evalúen las características de las ampolletas de vidrio respecto a su apariencia en el acabado y el color. Además, de que estén libre de defectos como grietas, asperezas, excesos de material y rebabas en el producto terminado. En el presente trabajo se desarrolló un prototipo de visión artificial automático, confiable y seguro para la inspección mecánica-visual, ordenamiento y empaquetamiento de ampolletas en la industria farmacéutica. Los elementos que componen el sistema son: un robot de la marca Mitsubishi RV-2FB-D, la controladora CR-750D, una cámara COGNEX, una banda transportadora, un motor de corriente directa y una ventosa neumática. Los resultados obtenidos en el proyecto permitieron validar el uso de la visión artificial para verificar dimensionalmente y visualmente ampolletas de color ámbar en un proceso automatizado, así como validar el ordenamiento de ampolletas en una misma posición dentro de su empaque.
\end{abstract}

Robot Mitsubishi RV-2FB-D, Vision artificial, Camara Cognex, Ampolletas

\begin{abstract}
One of the problems currently faced by the pharmaceutical industry in an automatic process, is to ensure that by means of a single traditional inspection system, the characteristics of the glass ampules are evaluated with respect to their appearance in the finish and the color. In addition to being free of defects such as cracks, roughness, excess material and burrs in the finished product. In the present work, a prototype of automatic artificial vision was developed, reliable and safe for the mechanical-visual inspection, ordering and packaging of ampoules in the pharmaceutical industry. The elements that make up the system are: a robot of the brand Mitsubishi RV-2FB-D, the controller CR750D, a camera COGNEX model ISM 1100-C11, a conveyor belt, a direct current (DC) motor and a vacuum suction cups, the latter responsible for taking the ampule. The results obtained in the project allowed to validate the use of artificial vision to visually and visually verify amber-colored ampoules in an automated process, as well as to validate the ordering of ampoules in the same position inside their packaging.
\end{abstract}

Robot Mitsubishi RV-2FB-D, Artificial vision, Cognex Camera, Ampule

Citación: GARCIA-GUTIEREZ, Felipe De Jesús, ROJAS-OLMEDO, Israel Alejandro, BARON- GUADARRAMA, Alma y SANCHEZ-MANCILLA, Luis Fernando. Inspección mecánico-visual de ampolletas farmacéuticas de vidrio mediante el uso de visión artificial y robótica. Revista de Ingeniería Innovativa. 2019. 3-10: 1-4

*Correspondencia al Autor (Correo electrónico: felipe.garcia@utvtol.edu.mx)

$\dagger$ Investigador contribuyendo como primer Autor. 


\section{Introducción}

En aplicaciones industriales el acabado superficial y las dimensiones de las piezas fabricadas son importantes para determinar su calidad debido a que estas se ensamblan con otras partes y tienen que acoplarse perfectamente. Las exigencias de fabricación de piezas de alta precisión y de calidad ha provocado que la industria en México cambie sus procesos de fabricación, de procesos manuales/semi-automáticos a procesos automatizados, y certifiquen sus procesos de fabricación contra la norma ISO 9000, requisito indispensable para exportar sus productos. Actualmente una forma de asegurar la calidad en el acabado de las ampolletas en la industria farmaceutica es a través de la inspección de las cotas críticas de la pieza y de los aspectos visuales importantes del acabado de la pieza, mediante el uso de algoritmos de visión artificial.

\section{Sistema de Visión Artificial (SVA)}

La visión artificial es la captación de imágenes en línea mediante cámaras y su posterior tratamiento a través de técnicas de procesamiento avanzadas, permitiendo así poder intervenir sobre un proceso (modificación de variables del mismo) o producto (detección de unidades defectuosas), para el control de calidad y seguridad de toda la producción [1]. El funcionamiento de un SVA se describe en cuatro pasos: El primero es la toma de imagen, el segundo el análisis de la imagen, el tercero envío de resultados y finalmente la toma de decisiones.

La inspección mecánico-visual de ampolletas farmacéuticas desarrollado en este trabajo se realizó de la siguiente manera, primero las piezas (ampolletas) arriban a la banda transportadora posteriormente el sensor detecta la pieza y envía una señal de disparo para posicionar al Robot Industrial Mitsubishi por encima del área de captura de la camara COGNEX, ésta adquiere la imagen de la ampolleta, los algoritmos del software se ejecutan en el sistema de visión realizando el procesamiento de la imagen y/o análisis de la imagen, sobre la imagen adquirida, la cámara envía una señal para posicionar el robot por encima de la ampolleta en buen estado, para posteriormente toma la pieza mediante una ventosa neumática, redireccional y la deposita en su empaque.

\section{Diseño del prototipo}

El prototipo estará conformado por el Robot RV2FB-D de Mitsubishi, la controladora CR-750D, una cámara de la marca COGNEX modelo ISM 1100-C11, una banda transportadora, un motor de corriente directa (CD) de la marca Baldor y una ventosa neumática, esta última encargada de tomar la ampolleta (ver figura 1). El software utlizado es In-Sight Explorer de COGNEX ${ }^{\mathrm{TM}}$, Melfa-Vision de MITSUBISHI ${ }^{\text {TM }}$ y RT Toolbox de MITSIBISHI.

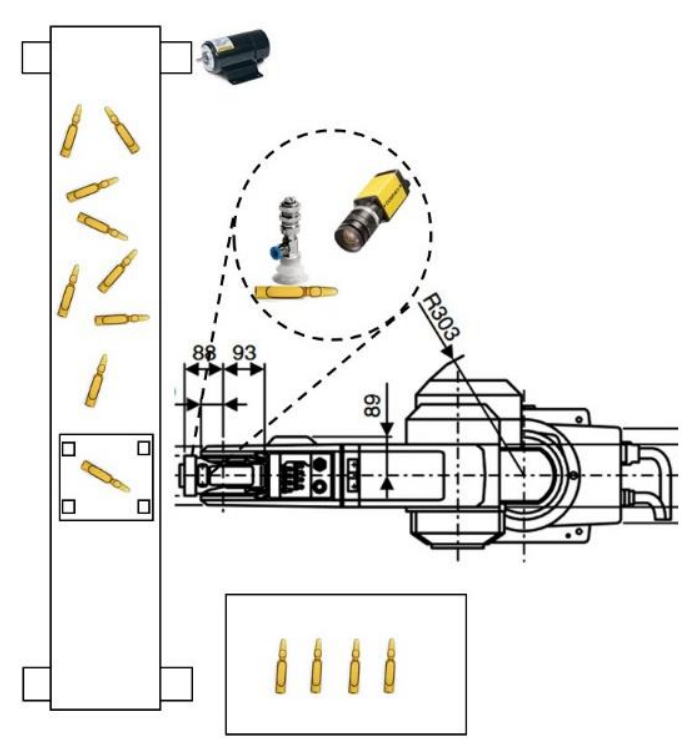

Figura 1 Diagrama esquemático del sistema

\section{Configuración y programación}

Para llevar a cabo la inspección mecánico-visual automática de ampolletas farmacéuticas, se usó la cámara marca COGNEX. La metodología que siguió fue la siguiente: Se configuraron los parámetros de la cámara y se calibró para la detección del área efectiva de detección, se sincronizó el robot con la cámara, para que esta última enviara la posición al robot, se programó una secuencia de posiciones para llevar la ampolleta en buen estado detectada para a su empaque.

\section{Resultados}

En la figura 2 se observa la integración de todos los elementos utilizados montados sobre una estructura de aluminio que limita el área de trabajo. Por otra parte, en la figura 3 se muestra con mayor detalle como se acoplo la cámara COGNEX, y la ventosa neumática al robot. La función de la ventosa es tomar la ampolleta después de su detección. 


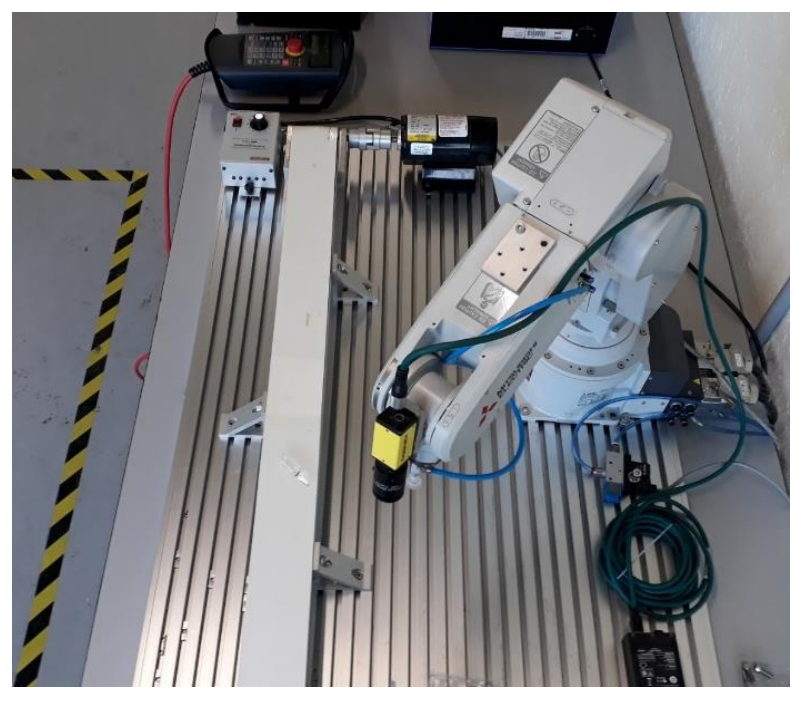

Figura 2 Ensamble de la cámara, robot y ventosa neumática
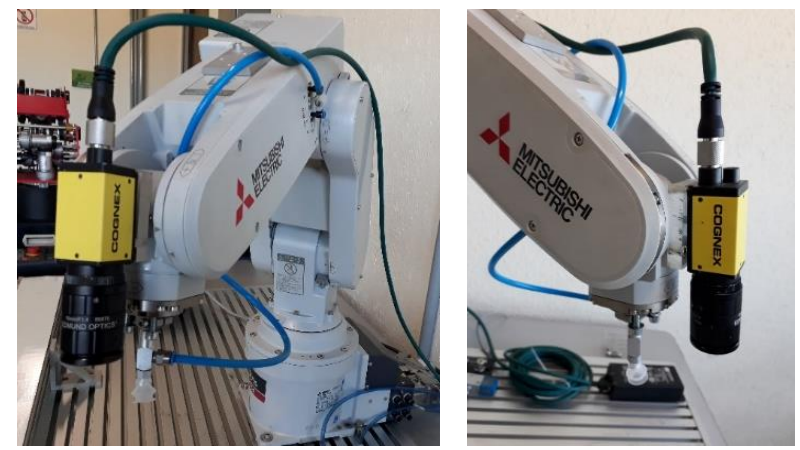

Figura 3 Detalle del ensamble de la cámara y la ventosa neumática

\section{Validación}

Como se puede observar en la secuencia de la figura 4, inicialmente el robot se posiciona en la parte superior del área de detección limitada por cuatro cuadrados de color negro, para después tomar la imagen y procesarla, como resultado se obtiene las coordenadas X, Y de la ampolleta dentro del área de detección, inmediatamente después el robot se posiciona en dichas coordenadas por encima de la ampolleta para después desplazare de forma vertical y tomarla mediante la ventosa neumática que es activada con una salida digital del puerto de la controladora. a)

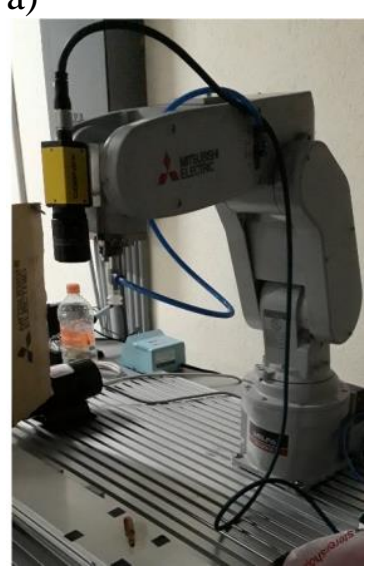

c)

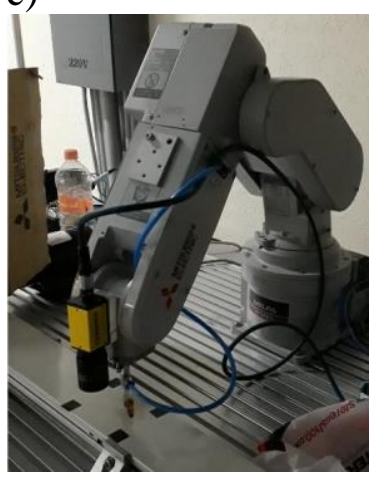

b)

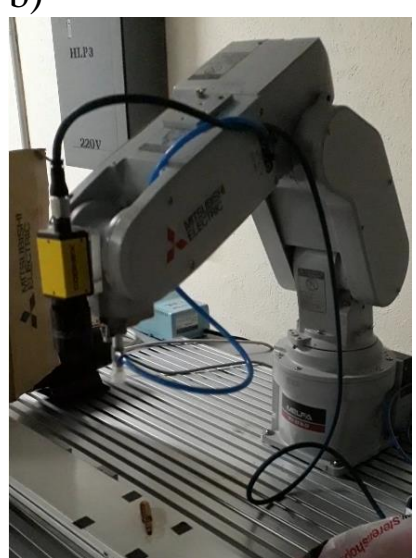

d)

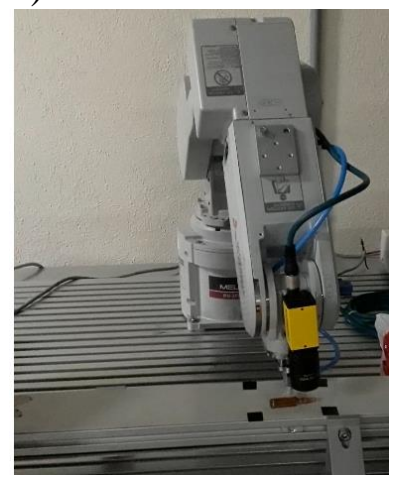

Figura 4 Secuencia de detección de la ampolleta

Después activar la ventosa neumática y tomar la ampolleta el robot gira lentamente ésta hasta ubicarla verticalmente como se muestra en las figuras $5 \mathrm{a}, 5 \mathrm{~b}$ y $5 \mathrm{c}$. Finalmente, el robot coloca la ampolleta en su empaque (ver figura $5 \mathrm{~d}$ ), un instante después regresa a la posición de captura justo encima del área de detección y envía la orden para poner nuevamente en marcha la banda transportadora y repetir el proceso.

a)

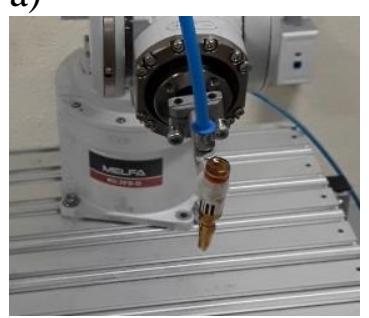

c)

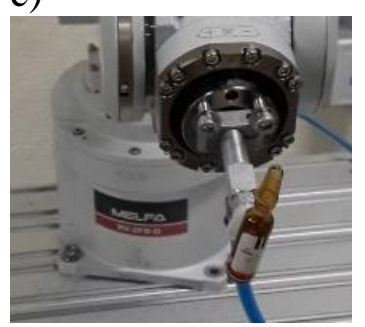

b)

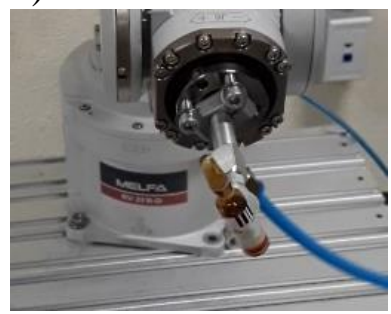

d)

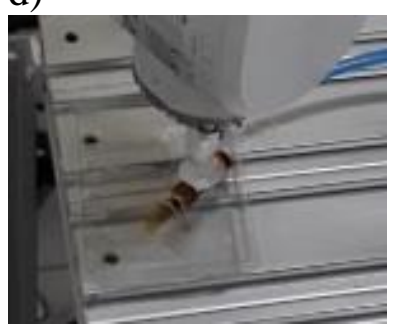

Figura 5 Secuencia de sujeción y colocación de la ampolleta en empaque 


\section{Agradecimiento}

El cuerpo académico de Mecatrónica de la Universidad Tecnológica del Valle de Toluca (UTVT) con clave UTVT-CA-5, agradecen Programa para el Desarrollo Profesional Docente, para el Tipo Superior (PRODEP), de la misma forma los autores agradecen a la UTVT, así como a la empresa Amats Electrics S.A de C.V. el desarrollo del presente trabajo.

\section{Conclusiones}

Sin duda uno de los grandes retos en los procesos automatizados es la inspección de partes a fin de asegurar la calidad de las mismas. El proyecto realizado permitió validar el uso de la visión artificial para verificar dimensionalmente y visualmente ampolletas de color ambar. Con los resultados obtenidos fue también posible validar el ordenamiento de ampolletas en una misma posición.

Sin embargo, uno de los problemas que se enfrentó durante el desarrollo del proyecto fue la detección de ampolletas de color transparente, debido a la refracción de la luz que no permitió tener una imagen nítida y en consecuencia un patrón común para reconocer los bordes de la ampolleta transparente y validar el acabado de la misma.

La correcta calibración del área efectiva permitió localizar las coordenadas correctas para que el efector final (ventosa) se posicionara justo en el centro de la ampolleta para tomarla, reorientarla y posteriormente colocarla en forma consecutiva dentro de un empaque. Como trabajo futuro se pretende incluir el paletizado de las ampolletas farmacéuticas, mejorar las condiciones de iluminación para que sea aplicable a ampolletas de color transparente

\section{Referencias}

Gobierno de Espa ña y Unión Europea. (2012). Visión Artificial Aplicación práctica de la visión artificial en el control de procesos industriales. Ministerio de Educación y Fondo Social Europeo, 26. 2018

Mitsubishi Electric (2010), MELFA Robots Industrial Robot Standard Specifications Manual RV-2SD/2SDB (CR1DA-700 series Controller), BFP- A8790-C, 136. 2018.
Mitsubishi. (2010). Network Vision Sensor Instruction Manual. Mitsubishi Industrial Robot (3D-51C-WINE，4D-2CG5100-PKG-E， 4D2CG5400-PKG-E，4D-2CG5401-PKG-E，4D2CG5403-PKG-E, 4D-2CG5400C-PKG-E, 4D2CG5400R-PKG-E, BFP-A8780), MELFA BFP-A8780-C, 166. 2018.

Mitsubishi. (2012). Standard Specifications Manual (CR2DA-701/CR3D-701M/CR3D-701 Controller). Mitsubishi Industrial Robot, MELFA BFP-A8656-Y, 168. 2018.

Obregón, L. K. G., Rincón, L. A. F., \& Suárez, O. M. D. (2018). Diseño e implementación de un sistema de visión artificial usando una técnica de mapeo y localización simultánea (SLAM) sobre una plataforma robótica móvil. Mundo FESC, 8(16), 8-17.

Rubio, C., Patricio, J., \& Díaz Quinga, J. R. (2018). Diseño e implementación de un prototipo de sistema flexible de almacenamiento/recuperación (AS/RS) automatizado mediante visión artificial para el reconocimiento dimensional de módulos de almacenamiento y optimización de espacios, para el Laboratorio de Mecatrónica en la Universidad de las Fuerzas Armadas ESPE extensión Latacunga (Bachelor's thesis, Universidad de las Fuerzas Armadas ESPE. Carrera de Ingeniería en Mecatrónica.). 